\title{
Stratifying Carotid Diseases for Endovascular Treatments
}

\author{
Gelin Xu Juehua Zhu Xinfeng Liu \\ Department of Neurology, Jinling Hospital, Nanjing University School of Medicine, \\ Nanjing, China
}

\section{Key Words}

Carotid artery $\cdot$ Atherosclerosis $\cdot$ Endovascular angioplasty and stenting $\cdot$ Individual therapy

\begin{abstract}
Endovascular angioplasty and stenting (CAS) has evolved as an alternative for treating occlusive carotid diseases in recent years. Carotid diseases are characterized by manifold etiologies and miscellaneous clinical manifestations. Although CAS is efficacious in treating patients with occlusive carotid diseases as a whole, the long-term risk-benefit ratio may vary in individual patients. Interventional strategies, such as angioplasty and stenting, should be individualized based on the etiology and clinical profiles to maximize the benefits and minimize the hazards of treatment. Based on recent publications, this review proposes a stratified treatment strategy for occlusive carotid diseases.

Copyright $\odot 2012$ S. Karger AG, Basel
\end{abstract}

Endovascular angioplasty and stenting (CAS) has emerged as an alternative for treating carotid artery diseases [1-5]. Patients with carotid diseases have manifold etiologies, such as arthrosclerosis, vasculitis, fibromuscular dysplasia (FMD), and artery dissection. The clinical outcomes of carotid diseases depend, to a considerable degree, on their etiologies. Diversities in angiography and clinical manifestations of carotid diseases are the reflections of heterogeneities in etiology and associated risk factors $[6,7]$. Therefore, interventional strategies should be weighed upon the clinical panorama of a given patient to maximize the benefits, and minimize the hazards, of intervention. This paper reviews possible influences on CAS based on recent progress.

Gelin Xu and Juehua Zhu contributed equally to this work. 


\section{Stratification of Clinical Symptoms}

As stroke risk is much higher in symptomatic carotid artery disease than in asymptomatic carotid disease [8], the presence of corresponding focal neurological symptoms is an essential factor determining the feasibility of intervention therapy. For a given patient, evaluation of the clinical symptoms, especially focal neurological deficits related to the target carotid artery disease, should include the severity, duration, frequency, and time of the initial symptoms. For symptomatic patients with $>50 \%$ carotid stenosis, CAS is recommended in the present guidelines, while it is less urgent and feasible for asymptomatic carotid disease. Although some guidelines recommend CAS in patients with $>70 \%$ asymptomatic carotid stenosis, the feasibility of CAS in patients with asymptomatic carotid stenosis has been challenged recently [9]. Several tools are available for quantitating the symptoms of stroke; the most frequently applied include the National Institutes of Health Stroke Scale (NIHSS) and the modified Rankin Scale (mRS). These scales have been used as references for selecting patients for intervention therapy and as tools for evaluating the outcomes of the procedures [10].

\section{Stratification of Etiologies}

Carotid diseases are associated with a variety of etiologies. By far, the evidence of CAS mainly comes from large-scale clinical trials of atherosclerotic carotid stenosis [1-5]. Indications for and contraindications of endovascular treatment have been made for this patient population. For carotid artery diseases related to other etiologies, such as vasculitis, FMD, artery dissection, radiation, and restenosis after surgery, no randomized controlled clinical trials are available due to the relatively low incidences of these diseases. The treatment choices of these patients have been referred to results from small sample observational studies or case reports. Currently, the mainstream for carotid disease of vasculitis etiology management is anti-inflammatory treatment such as steroids. If patients present with focal neurological symptoms, especially with evidence of clinical deterioration and progressive carotid stenosis independent of anti-inflammatory treatment, CAS may be considered [11]. However, there is evidence that CAS may deteriorate the local vascular inflammatory response and lead to restenosis or occlusion of the treated artery [12]. For patients with carotid FMD, results from observational studies have indicated relatively favorable outcomes of CAS [13]. The most common presentation of carotid FMD is transient ischemic attack or stroke. In FMD with repeated ischemic cerebral events, limited data indicate that CAS could arrest the recurrent events in selected patients. It is noteworthy that, due to the vessel wall abnormalities in FMD, percutaneous balloon angioplasty alone might not be effective enough and stenting is usually required for maintaining the normal lumen of carotid in FMD patients [14].

Carotid artery dissection (CAD) is an underdiagnosed but significant cause of stroke. Patients with CAD in general have favorable prognoses with conservative therapy, such as anticoagulants. However, if there exists a large dissection or it is accompanied by an obvious aneurysm, or there is marked expansion of the dissection proximally or distally, aggressive intervention may be justified [15]. Notably, all CAD patients, treated with or without CAS, are supposed to receive efficient anticoagulation to prevent acute intravascular thrombosis [16]. Open surgery is rarely considered for radiation-induced carotid stenosis and restenosis after carotid endarterectomy. However, among those with corresponding local neurological symptoms, CAS may be indicated $[5,17]$. 


\section{Assessment and Stratification of Stenosis Severity}

Assessment methods for carotid artery disease include digital subtraction angiography (DSA), magnetic resonance angiography (MRA), computerized tomographic angiography (CTA), cervical vascular ultrasound, and transcranial Doppler ultrasonography (TCD). Among them, DSA, MRA, and CTA are most commonly used due to their reliability and validity. TCD was recommended by the 2011 American Heart Association (AHA) guidelines for secondary prevention of stroke owing to its easy operation, noninvasiveness, and low cost [18]. The severity of carotid artery disease, especially the degree of stenosis, is an important factor affecting the strategy of intervention therapy. Therefore, this indicator requires accurate measurement. Currently, the NASCET method is most frequently used to measure the degree of carotid stenosis [19]. The mainstream view is that CAS is indicated in symptomatic atherosclerotic carotid artery disease with stenoses of $>50 \%$ [16] and asymptomatic atherosclerotic carotid artery disease with stenoses of $>70 \%$ [20]. For carotid diseases caused by etiologies other than atherosclerosis, due to the lack of evidence from randomized clinical trials, criteria of stenosis degree for intervention therapy, if need, are referred to that of atherosclerotic etiology.

\section{Assessment of Endovascular Access}

Unlike carotid endarterectomy which accesses the focus via open surgery, endovascular therapy accesses the artery lesion via the vascular lumen. Therefore, the technical success of an endovascular procedure depends largely on appropriate access. Possible factors affecting endovascular access include tortuosity, kinking, aneurysm, dissection, and stenosis in proximal arteries (femoral, iliac, subclavian, carotid arteries, and aorta), type III aortic arch, and complicated carotid lesions (severe calcification, long lesion, and high-degree stenosis). Stenosis, tortuosity, and kinking in the distal artery may influence the landing of the brain protection device and maneuvering of the wire and catheter. Therefore, extra attention should be paid to these factors. Endovascular procedures may deteriorate the aneurysm or dissection in the distal artery, and even lead to aneurysm rupture, with fluctuation of blood pressure, heparinization, and mechanical injury during the procedure as possible causes. In this circumstance, the common understanding is that the distal aneurysm and dissection should be treated, if possible, before the CAS. Currently, most CAS procedures use the right femoral artery as the access. If this access is not applicable, the left femoral artery, brachial artery, or carotid artery could be an alternative [21].

\section{Assessment of Plaque Stability}

Atherosclerotic disease is characterized by artery plaque formation in advanced lesions. An important mechanism of cerebral infarction is distal artery embolization by debris from the broken atherosclerotic plaque. The broken atherosclerotic plaques may expose sub-intima tissues to circulation, which triggers and promotes intraluminal thrombosis and consequently causes cerebral infarction. Based on the risk of rupture and the course of the diseases, atherosclerotic plaques were classified as stable or vulnerable. Theoretically, patients with vulnerable plaque should be treated with aggressive interventions because of their high risk of plaque rupture and the high incidence of ischemic cerebrovascular events. On the other hand, patients with stable plaque may be deferred with CAS and treated medicinally due to their low risk of subsequent cerebrovascular events. Another advantage of classifying 
plaque vulnerability lies in that this strategy may grossly predict the risk of periprocedural complications, because the vulnerable plaque is more prone to break and cause retina or cerebral infarction during the procedure. Therefore, patients with vulnerable plaque should be handled with extra caution during the endovascular procedures. Unfortunately, to date there are no reliable criteria for defining stable and vulnerable plaques, and the feasibility of a few measures used to distinguish vulnerable plaque from stable plaque are controversial.

When assessing the vulnerability, or stability, of a plaque, clinical manifestation, neuroimaging, and angiographic results are worthy of consideration. If patients present with recurrent ischemic events (stroke or transient ischemic attack) which can be associated with the target lesion, vulnerable plaque should be highly suspected regardless of the results of neuroimaging and angiographic examinations.

Many examinations have been designed to evaluate the stability of plaque, although few have been proven effective. DSA permits dynamic observation of regional blood flow near the lesion and can provide information on plaque morphology, such as ulcers, and internal plaque bleeding.

The advantages of CTA include its sensitivity in identifying calcification of the plaque and evaluating the eccentricity of stenosis. Calcification has been accepted as an indication of plaque vulnerability. Target lesions with prominent calcification may limit the passage of guide wire and brain protection devices and consequently decrease the technical success rate of CAS procedures. Severe calcification may also resist balloon angioplasty and encumber stent deployment, both of which may increase the degree of residual stenosis after CAS.

Ultrasound is also suggested as a simple tool for evaluating plaque vulnerability. Echorich plaques are usually regarded as stable, while echolucent plaques are considered vulnerable. The risk of subsequent stroke is significantly higher in patients with echolucent carotid plaques than in patients with echorich plaques [22]. Recently, new techniques such as intravascular ultrasound and optical coherence tomography (OCT) have been attempted in evaluating the vulnerability of atherosclerotic plaques $[23,24]$. Intravascular ultrasound is an imaging methodology which uses a specially designed catheter with a miniaturized ultrasound probe attached to the distal end of the catheter. The proximal end of the catheter is attached to computerized ultrasound equipment. It allows the application of ultrasound technology to see from inside the blood vessels out through the surrounding blood column, thereby visualizing the endothelium of the artery. OCT is an optical signal acquisition and processing method. It captures micrometer resolution and three-dimensional images from within optical scattering media. Researchers are evaluating the feasibility of frequency domain OCT in detecting vulnerable lipid-rich plaques.

Other researchers have suggested that fMRI and PET could be used to evaluate the stability of atherosclerotic plaque [25]. The rationality of this novel application lies in that active atherosclerotic lesions in vulnerable plaques can express high-level inflammatory cytokines and may result in increased regional blood flow and an elevated metabolic rate, which can be detected by fMRI and PET, respectively. However, due to several unsolved technical problems, clinical application of these new strategies has not been commenced.

\section{Assessment of Cerebral Collateral Circulation}

Collateral circulation may function by the formation of new vessels (angiogenesis) or reopening of the fetal anastomoses (the persistent trigeminal, otic, hypoglossal, and proatlantal intersegmental arteries), or via the circle of Willis. Collateral circulation may play an important role in patients with carotid stenosis or occlusion, which may attenuate or reverse ischemic brain injury. In patients with potent collaterals, even if the carotid has severe ste- 
nosis or occlusion, the clinical symptoms may be mild or absent. Studies have observed that patients with abundant collaterals have a decreased risk of subsequent stroke compared with patients without them. This phenomenon is understandable because abundant collaterals mean decreased blood flow and weakened shear stress for carotid plaques; as a result the risk of plaque rupture decreases. Well-formed collateral circulations also compensate hypoperfusion associated with occlusive carotid lesions and, therefore, decrease the risk of watershed cerebral infarctions. For patients without efficacious collaterals, less severe carotid stenosis may lead to stroke occurrence or recurrence. Theoretically, these patients with poor or no collaterals pose a priority for CAS treatment. DSA is an expedient tool to assess collateral status in patients with carotid stenosis. Other means such a MRA, CTA, TCD, and ultrasound may be helpful. Collateral circulation may also be evaluated indirectly by measuring the local cerebral blood flow and tissue metabolic rate with SPECT and PET, respectively. A low metabolism rate in the brain regions irrigated by the target carotid artery might be an indication for aggressive intervention [26].

\section{Assessment of Patient Factors and Comorbidities}

Many patients with atherosclerotic carotid diseases have one or more chronic comorbidities as atherosclerotic risk factors. Comorbidities should be evaluated and treated properly, if possible, before CAS is performed. Long-term hypertension might lead to cardiac and renal function deficiency, which decreases the tolerance to contrast agent toxicity and increases the incidence of induced hemodynamic reactions and cardiovascular events to mechanical stimulations. Chronic hypertension may result in heart enlargement and uplift of the aortic arch, artery elongation and stiffness, all of which may consequently cause tortuosity and kinking of the aortic arch and carotid arteries, challenging the successfulness of CAS procedures. Chronic hyperglycemia impairs the vascular endothelium, increases the vascular fragility and instability of atherosclerotic plaque, and consequently increases the risk of CAS procedures. To prevent intraluminal thrombosis and restenosis, heparinization is usually applied during CAS and antiplatelet therapy prescribed after the CAS procedures. From the medication perspective, blood system diseases, thrombocytopenia, bleeding tendency, a recent major operation, or an upcoming major operation are contraindications for CAS [18, 20].

\section{Assessment of the Socioeconomic Status of Patients}

Compared with traditional medication, endovascular intervention is relatively expensive, so the choice of treatment should be weighed against the economic status of the patients, especially those in developing countries. In addition, after CAS, long-term antiplatelet drugs and regular clinic examinations are required. Therefore, the assessment and availability of long-term antiplatelet treatment should be considered when making the treatment plan.

In summary, CAS is a proven treatment for carotid artery disease. However, this procedure is not suitable for all patients with carotid diseases. When making the treatment plan, the above factors should be evaluated and managed adequately before CAS. Only stratified and individualized treatment programs can minimize the risk and maximize the benefits of this challenging intervention. 


\section{References}

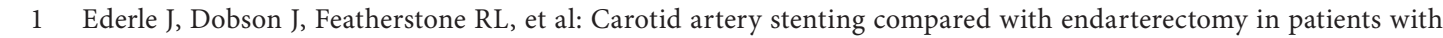
symptomatic carotid stenosis (International Carotid Stenting Study): an interim analysis of a randomised controlled trial. Lancet 2010;375:985-997.

-2 Brott TG, Hobson RW 2nd, Howard G, et al: Stenting versus endarterectomy for treatment of carotid-artery stenosis. N Engl J Med 2010;363:11-23.

-3 Bonati LH, Dobson J, Algra A, et al: Short-term outcome after stenting versus endarterectomy for symptomatic carotid stenosis: a preplanned meta-analysis of individual patient data. Lancet 2010;376:1062-1073.

4 Ringleb PA, Allenberg J, Bruckmann H, et al: 30 day results from the SPACE trial of stent-protected angioplasty versus carotid endarterectomy in symptomatic patients: a randomised non-inferiority trial. Lancet 2006;368:1239-1247.

5 Yadav JS, Wholey MH, Kuntz RE, et al: Protected carotid-artery stenting versus endarterectomy in high-risk patients. N Engl J Med 2004;351:1493-1501.

6 Sitzer M: Atherosclerotic carotid stenosis and occlusion. Front Neurol Neurosci 2006;21:36-56.

7 Cohen JE, Itshayek E: Asymptomatic carotid stenosis: natural history versus therapy. Isr Med Assoc J 2010;12:237-242.

8 Rothwell PM: Endarterectomy for symptomatic and asymptomatic carotid stenosis. Neurol Clin 2008;26:1079-1097.

-9 Broderick JP: The challenges of intracranial revascularization for stroke prevention. N Engl J Med 2011;365:10541055.

10 Choi BS, Park JW, Shin JE, Lü PH, Kim JK, Kim SJ, Lee DH, Kim JS, Kim HJ, Suh DC: Outcome evaluation of carotid stenting in high-risk patients with symptomatic carotid near occlusion. Interv Neuroradiol 2010;16:309-316.

11 Us M, Numan F, Goksel OS, et al: Stenting for stenosing Takayasu aortitis following carotid artery stenosis in a 32-year-old patient. Vascular 2008;16:283-286.

12 Lee BB, Laredo J, Neville R, et al: Endovascular management of Takayasu arteritis: is it a durable option? Vascular 2009;17:138-146.

13 Olin JW, Sealove BA: Diagnosis, management, and future developments of fibromuscular dysplasia. J Vasc Surg 2011; 53:826-836.e1.

14 Finsterer J, Strassegger J, Haymerle A, et al: Bilateral stenting of symptomatic and asymptomatic internal carotid artery stenosis due to fibromuscular dysplasia. J Neurol Neurosurg Psychiatry 2000;69:683-686.

15 Ohta H, Natarajan SK, Hauck EF, et al: Endovascular stent therapy for extracranial and intracranial carotid artery dissection: single-center experience. J Neurosurg 2011;115:91-100.

16 Jeon P, Kim BM, Kim DI, et al: Emergent self-expanding stent placement for acute intracranial or extracranial internal carotid artery dissection with significant hemodynamic insufficiency. AJNR Am J Neuroradiol 2010;31:15291532 .

17 Tallarita T, Oderich GS, Lanzino G, et al: Outcomes of carotid artery stenting versus historical surgical controls for radiation-induced carotid stenosis. J Vasc Surg 2011;53:629-636.e1-5.

18 Furie KL, Kasner SE, Adams RJ, et al: Guidelines for the prevention of stroke in patients with stroke or transient ischemic attack: a guideline for healthcare professionals from the American Heart Association/American Stroke Association. Stroke 2011;42:227-276.

19 Beneficial effect of carotid endarterectomy in symptomatic patients with high-grade carotid stenosis - North American Symptomatic Carotid Endarterectomy Trial Collaborators. N Engl J Med 1991;325:445-453.

20 Goldstein LB, Bushnell CD, Adams RJ, et al: Guidelines for the primary prevention of stroke: a guideline for healthcare professionals from the American Heart Association/American Stroke Association. Stroke 2011;42:517-584.

21 Bakoyiannis C, Economopoulos KP, Georgopoulos S, et al: Transradial access for carotid artery stenting: a singlecenter experience. Int Angiol 2010;29:41-46.

22 Grønholdt ML, Nordestgaard BG, Schroeder TV, Vorstrup S, Sillesen H: Ultrasonic echolucent carotid plaques predict future strokes. Circulation 2001;104:68-73.

23 Politi L, Aprile A, Rollini F, Amato A, Biondi-Zoccai G, Mauriello A, Modena MG, Sangiorgi GM: Carotid plaque characterisation by IVUS-VH during carotid stenting: the 'eyes wide shut' between plaque morphology and symptoms. Minerva Cardioangiol 2011;59:591-600.

-24 Reimers B, Nikas D, Stabile E, Favero L, Saccà S, Cremonesi A, Rubino P: Preliminary experience with optical coherence tomography imaging to evaluate carotid artery stents: safety, feasibility and techniques. EuroIntervention 2011; 7:98-105.

25 Hermus L, van Dam GM, Zeebregts CJ: Advanced carotid plaque imaging. Eur J Vasc Endovasc Surg 2010;39:125-133.

26 Chaer RA, Shen J, Rao A, et al: Cerebral reserve is decreased in elderly patients with carotid stenosis. J Vasc Surg 2010; 52:569-574, discussion 574-565. 